\title{
Applicability of DSDV and DSR Routing Protocol for MANET with Mobile Gateway
}

\author{
Abu Bony Amin \\ Department of Electrical \& Electronic Engineering, \\ Chittagong University of Engineering and \\ Technology (CUET), Chittagong-4349, \\ Bangladesh
}

\author{
Choton Kanti Das \\ Department of Electrical \& Electronic Engineering, \\ Chittagong University of Engineering and \\ Technology (CUET), Chittagong-4349, \\ Bangladesh
}

\begin{abstract}
In this technological era mobile communication among air transportation is increasing day by day. Maintaining seamless communication by avoiding congestion in a cost effective way is a future challenge. This future challenge can be met by developing solutions for an aeronautical air-ground communication network based on internet technologies. This is done by developing Mobile Ad hoc Network (MANET). Mobile Ad hoc networks (MANETs) are infrastructure-less dynamic networks allowing mobile nodes to communicate beyond transmission range. Nodes in a MANET that connect it to the Internet are called Internet gateways. This research will present a new paradigm for aeronautical communication in air to ground and air to air at future with mobile gateway. Most of the previous researches on MANET were focused on static gateway. In this paper two routing protocols DSDV (Destination Sequenced Distance Vector) and DSR (Dynamic Source Routing Protocol) are considered for simulation by Network Simulator (NS-2.35) and shown by performance metrics which is the best one.
\end{abstract}

\section{Keywords}

Destination Sequenced Distance Vector (DSDV), Dynamic Source Routing Protocol (DSR), MANET, mobile gateway, Network Simulator (NS-2.35).

\section{INTRODUCTION}

Mobile communications and wireless internet access are increasingly becoming an essential part of people's lives in this era of information technology. The growing interest by commercial, rescuer and fighter aircrafts in providing internet access and cellular connectivity in the cabin has led to the emergence. Apparently, a satellite communications link is the most feasible and flexible way to keep the aircraft connected to the ground throughout the flight. But with a satellite, there is always a one-way end-to-end propagation delay required for the signal to travel up and down from the satellite. This is not cost effective for bandwidth usage. So, other effective way can be applied to obtain seamless communication such like as Mobile Ad hoc network [1].

A Mobile Ad hoc Network (MANET) is a collection of wireless nodes that can dynamically form a network to exchange information without using any pre-existing fixed network infrastructure. Moreover, MANET is a self-organized and self-configurable network where the mobile nodes move arbitrarily. The mobile nodes can receive and forward packets as a router. Each node operates not only as an end system, but also as a router to forward packets. The nodes are free to move about and organize themselves into a network. These nodes change position frequently. A pair of mobile nodes may communicate with each other either directly or indirectly with the help of the intermediate nodes in the Mobile Ad Hoc Network [2][3][4]. Most of the previous researches on MANET with different routing protocols have mainly analysed the horizontal and vertical scenario in a communication environment [5]. But in this approach an aircraft will act as a gateway. So, in this research gateway is configured as mobile by which this approach can be applied in the aeronautical communication. It is our a very little attempt to obtain a cost effective way to communicate in air to air without using most costly Satellite link.

\section{SIMULATION SCENARIO DESCRIPTION}

Since, in the MANET the aircrafts themselves act as a gateway therefore, the simulation is done for mobile gateway. Here by this network air-ground as well as air-air communication can be made very effectively. The usage of Satellite link will be reduced. So, the end-to-end delay will be reduced appreciably. Consequently, a cost effective way for communication will be achieved.

Fig 1 shows that MANET is envisioned as a means to extend the coverage of air-ground access networks. By enabling aircraft themselves to act as network routers, a mobile ad hoc network is formed in the sky.

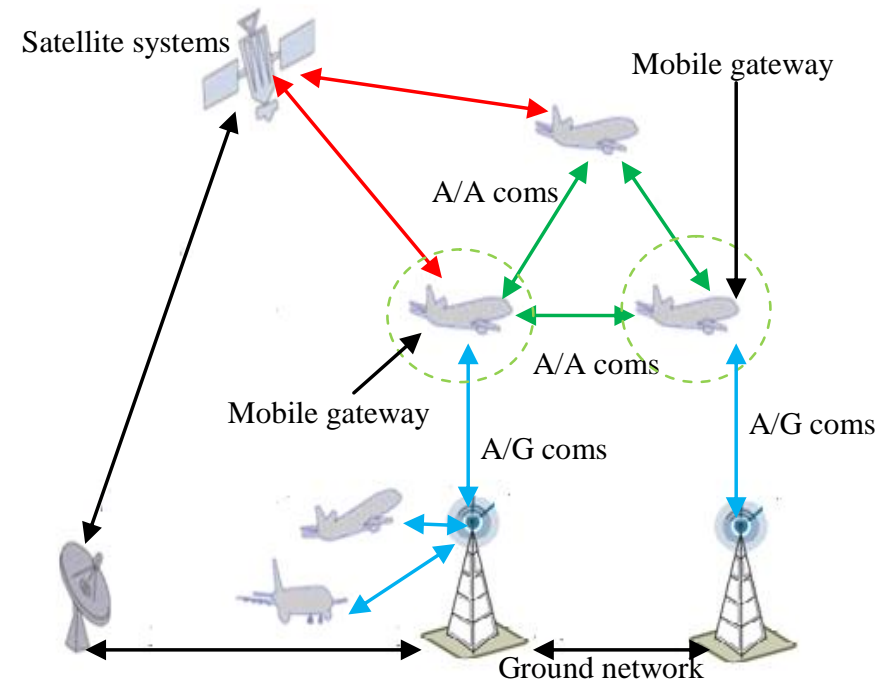

Fig 1: Scenario of air-ground (A/G coms) and air-air (A/A coms) communication by Mobile Ad hoc Network with mobile gateways [6]. 
Fig 1 shows that MANET is envisioned as a means to extend the coverage of air-ground access networks. By enabling aircraft themselves to act as network routers, a mobile ad hoc network is formed in the sky.

\section{SIMULATION ARRANGEMENTS}

The network performances such as throughput, packets dropped, packet delivery ratio, end-to-end delay, packets received and packets forwarded were evaluated using NS-2 simulator. The entire simulations were accomplished by using NS-2.35 network simulator which is a discrete event driven simulator [7]. This section describes the network scenario, the movement model, the communication model, and the simulation parameters that used in the network topology.

\subsection{Network Architecture}

The simulated network is spanning in a standard area of $1000 \times 1000 \mathrm{~m}^{2}$. Each mobile node in our simulation has a wireless transmission range of 250 meter, which is the standard range and also used by the other research works [8]. In this simulation scenario 2 gateways are considered in the MANET in order to load balance the Internet traffic. Both of the gateways are configured as mobile gateway. A higher Internet bandwidth for gateways compared to that of the MANET nodes is assumed. Simulations are run for 200 units of simulation time. A screenshot of a simulation scenario is given in Fig 2. In the figure, the green colored circular node represents the gateways, whose node id is 14 . The node 7 is also marked as gateway because, it was acting as a gateway at previous operation. Both the gateways are mobile. Node 1 and node 13 act as source and destination respectively. In this scenario, Node 4, 5, 6, 7, 14 are configured as mobile nodes.

In this network topology the number of nodes is kept constant but the mobility of nodes is varied for different routing protocols.



Fig 2: Screenshot of a simulation scenario in NS-2 NAM window.

\subsection{Movement Model}

The Random Waypoint is deactivated in the Movement Model and the velocity and coordinate of node as the mobility model are manually defined for the simulation. In this model, mobile node remains stationary for a certain period called pause time. When the pause time is over, the node moves toward the defined destination at a defined speed. The different types of speeds such as $0,5,10,15,20,25 \mathrm{~m} / \mathrm{s}$ are set for different scenarios. When the node reaches the destination, it again remains stationary for the pause time period and repeats the same procedure until the end of the simulation. The pause time is set to 20 seconds in our simulations which is good enough for a node to change the movement direction.

\subsection{Communication Model}

Each mobile node in this simulation uses Transmission Control Protocol (TCP) traffic to send packets to the corresponding hosts in the Internet. Each mobile node is permitted to generate packets of size 512 bytes in the MANET. By varying the velocity of nodes, the characteristics of different routing protocol are actually observed in Mobile Ad Hoc Network with different simulation scenarios.

\subsection{Mobility Generation Model}

The movement scenario can be defined for each simulation. To study the effect of mobility, the simulation is carried out with movement patterns generated for different velocity of mobile nodes. In this simulation, the velocity of nodes is defined as $0,5,10,15,20,25 \mathrm{~m} / \mathrm{s}$ respectively. The "setdest" command of NS-2 simulators is used to generate nodemovement. By using "setdest" the next destination of the mobile node and its velocity can be defined manually.

\subsection{Parametric Quantities}

Table I gives the values of simulation parameters that are used for the simulation scenarios.

Table 1. Common parametric quantities used in the simulation scenarios [9]

\begin{tabular}{|c|c|}
\hline Parametric quantities & Value \\
\hline Number of nodes & 15 \\
\hline Number of gateways & 2 \\
\hline Number of hosts & 2 \\
\hline Number of mobile nodes & 11 \\
\hline Topology size & $1000 \times 1000 \mathrm{~m}^{2}$ \\
\hline Transmission range & $250 \mathrm{~m}$ \\
\hline Channel type & Wireless \\
\hline MAC type & 802.11 \\
\hline Traffic type & TCP \\
\hline Packet size & 512 bytes \\
\hline Routing protocol & DSDV/DSR \\
\hline Pause time & $20 \mathrm{~s}$ \\
\hline Simulation time & $200 \mathrm{~s}$ \\
\hline
\end{tabular}

\section{SIMULATION PARTICULARS}

In this section, the gateway discovery and packet transmission procedure will be shown. When the node speed is set to $5 \mathrm{~m} / \mathrm{s}$ for DSDV routing protocol firstly the source discover the node 7 as a gateway. But node 7 was a mobile gateway. It moved away from the source. So, the source lost the gateway. 
At that time, the node 7 dropped the packets which are sent from node 1. Fig 3 depicts this occurrence.



Fig 3: Screenshot of a NAM window for node speed $5 \mathrm{~m} / \mathrm{s}$ when node 7 acts as a gateway

After losing node 7 as a gateway the node 1 is searching for new gateway for sending packet to the destination node 13. But at this time only some node will advertise itself to the node 1 which is shown in Fig 4. It is noticeable from Fig 4 that, node 8 and node 9 are not advertising themselves to act as a gateway. It is for the DSDV routing protocol.

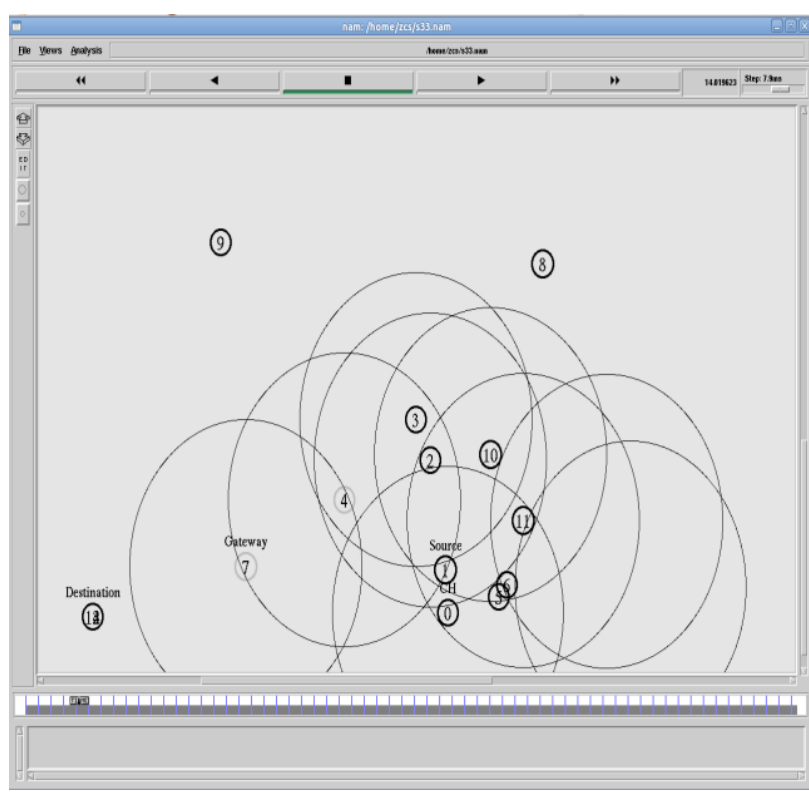

Fig 4: Screenshot of a NAM window for node speed $5 \mathrm{~m} / \mathrm{s}$ when node 1 searching for gateway

Fig 5 depicts that when node 14 is not ready to become a gateway, the former gateway node 7 is starting to drop the packet which are sent from the source node1. So the packet losses have occurred there. But, if the velocity of node 14 is increased, then the amount of packet loss is decreases.

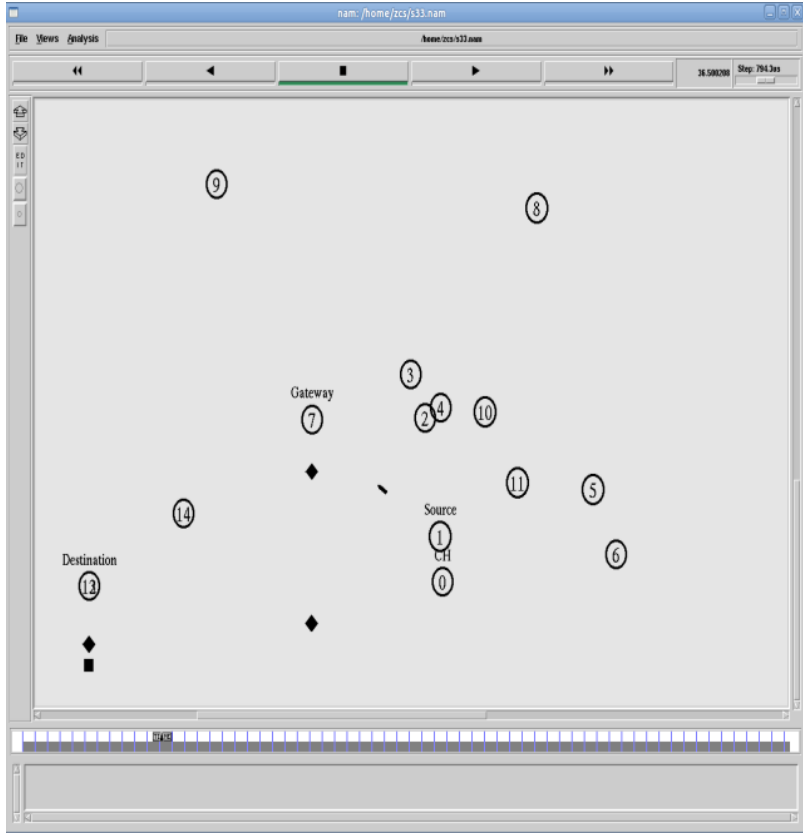

Fig 5: Screenshot of a NAM window for node speed $5 \mathrm{~m} / \mathrm{s}$ when node 7 drops the packets

At last, the node 14 moves toward the source node slowly. So, the source node gets the node 14 at its sight which is shown in Fig 6. In this scenario, finally the node 14 is selected as gateway. So the packet transfer is performed from source node 1 to destination node 13 via the gateway node 14 .

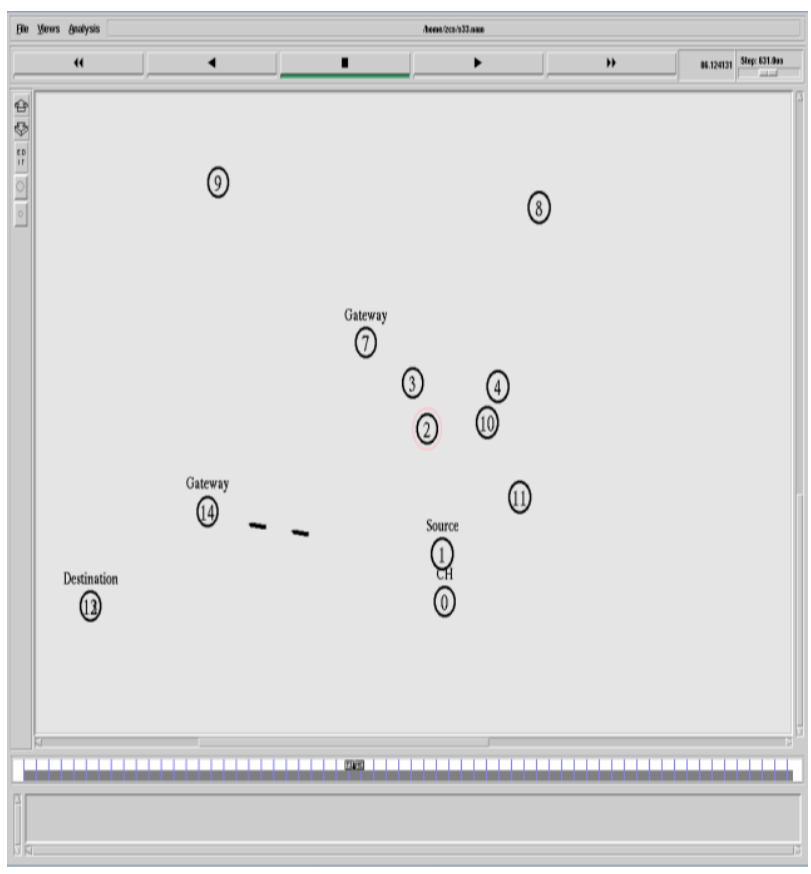

Fig 6: Screenshot of a Nam window for node speed $5 \mathrm{~m} / \mathrm{s}$ when node 14 selected as a gateway

\section{SIMULATION OUTCOMES}

The simulation outcomes are shown in the following section in the form of line graphs. From Fig 7 to Fig 11 represent the graphs which demonstrate the comparison between the two protocols. 


\subsection{Throughput}

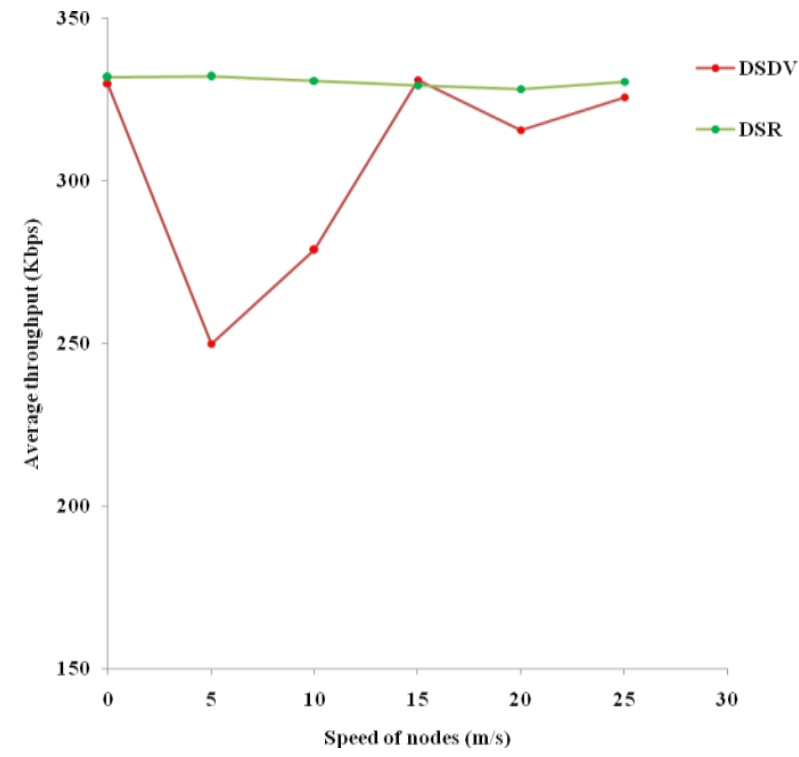

Fig 7: Average throughput vs. Speed of nodes for different routing protocol

Fig 7 depicts that, throughput of DSDV is very poor at lower mobility; hence performance of DSDV protocol increases as mobility increases compared DSR protocol. The throughput of DSR is near to same for any mobility. So, for higher mobility performance of the DSDV protocol is better.

\subsection{Packet Delivery Ratio(r/s)}



Fig 8: Packet Delivery Ratio vs. Speed of nodes for different routing protocol

It is clear from Fig 8 that, the packet delivery ratio of DSR is very less as compared to on demand protocol DSR at higher mobility. DSDV perform best among all at high mobility. The reason for having better packet delivery ratio of DSDV is that it allows packets to stay in the send buffer for route discovery and once the route is discovered, data packets are sent on that route to be delivered at the destination.

\subsection{End-to-End Delay}

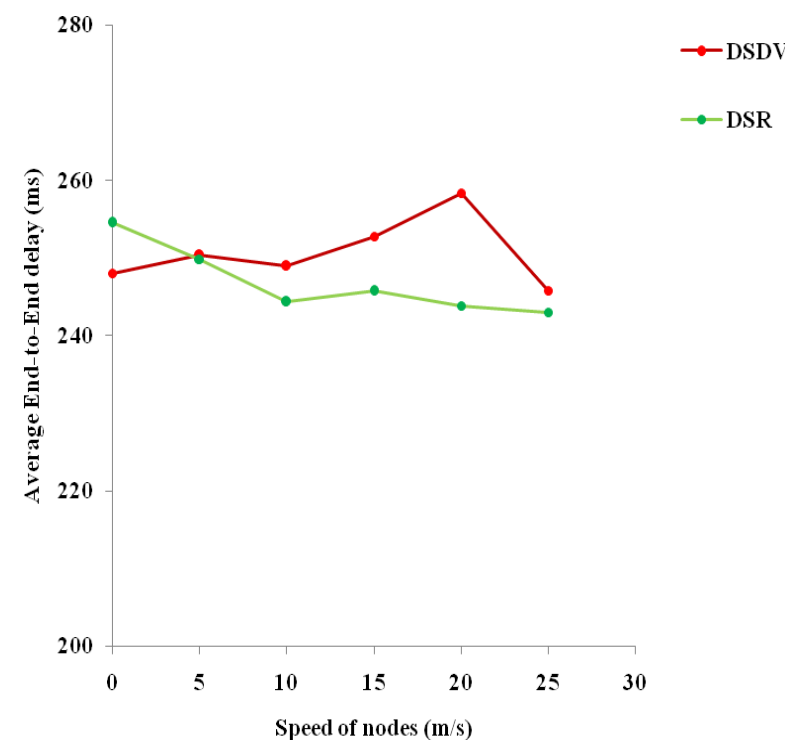

Fig 9: Average End-to-End delay vs. Speed of nodes for different routing protocol

End-to-End delay in DSDV is better compared to DSR as shown in Fig 9. Because, DSDV always holds the optimal paths to destinations in their routing table and hence it can send data packets immediately. However, high end-to-end delay can be justified as DSR delivers more packets at the destination as compared to DSDV because this protocol tries to provide some sort of guarantee for the packets to be delivered at the destination by compromising at the cost of delay.

\subsection{Packets Received}

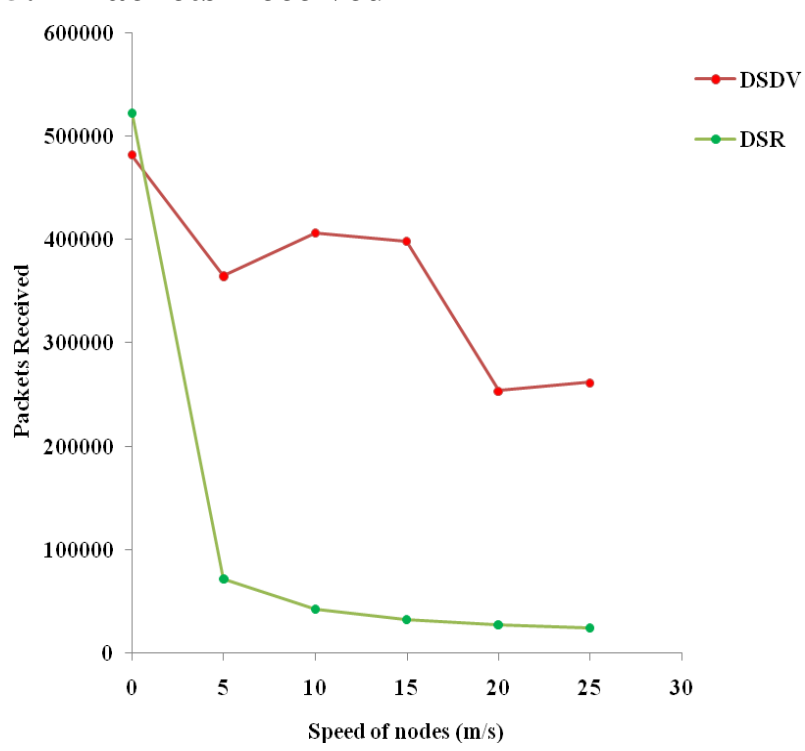

Fig 10: Packets received vs. Speed of nodes for different routing protocol

It is noticeable from Fig 10 that, the amount of packet received in DSDV is better compared to DSR. It is shown from the graph that the amount of packet received is decreases with increasing mobility of nodes for both DSDV and DSR. 
Even for DSR the amount of packet received decreases abruptly with increasing mobility of nodes.

So, the performance of DSDV routing protocol is better than DSR routing protocol for packet received.

\subsection{Packets Forward}



Fig 11: Packets sent vs. Speed of nodes for different routing protocol

For packet forward performance DSDV is better than DSDV which is shown in Fig 11. For both DSDV and DSR the packet forward decreases with increasing the velocity of the mobile nodes.

The overall conclusion is that, for a MANET with mobile gateway, DSDV is better choice with better performance than DSR.

\section{USEFULNESS OF THE APPROACH}

By using this Mobile Ad hoc Network with mobile gateway a seamless air to ground communication can be established which will be cost effective because there have no need to any base station and infrastructure. The Mobile Ad Hoc Network is very useful to establish a seamless communication in disaster area to rescue purpose [10]. The effectiveness in usage of bandwidth will appreciably increase. Opportunities for better information availability and sharing at anywhere and anytime will increase. By adopting the best routing protocol for this network the most reliable communication network can be built. This type of network is also eligible for air to air communication without any range limitations.

\section{CONCLUSIONS}

Nowadays, the air traffic is increasing rapidly. So, new technological solutions for avionics have to be developed to meet the heavily loaded Internet traffic. Cost savings and operational improvements through better and greater access to aircraft data while on ground can be met by wireless broadband access to aircraft and flight data upon landing during turnarounds and layovers and use of low cost wireless technology to transfer large amounts of non-critical data. To meet the future requirements for robust aeronautical communication network, interface among different types of communication network making is very important for which the aeronautical mobile Ad hoc network have to be adapted. In this approach, it was already shown by considering various performance metrics that the performance of aeronautical communication network is improved when the mobile Ad hoc network is used. So, the advanced research work on aeronautical Mobile Ad hoc Network with heavily loaded gateway by the Internet traffic can make a perfect robust aeronautical communication network.

\section{REFERENCES}

[1] D. Medina and F. Hoffmann, "The Airborne Internet" Future Aeronautical Communications, Edited by S. Plass, September 2011.

[2] P. Sharma, A. Kalia and J. Thakur, " Performance analysis of AODV, DSR AND DSDV routing protocols in mobile ad-hoc network (MANET)" Journal of Information Systems and Communication, Bioinfo Publications, Volume 3, Issue 1, 2012, pages: 322-326.

[3] I. Chlamtac, M. Conti, J. J.-N. Liu, "Mobile ad hoc networking: imperatives and challenges" Ad Hoc Networks, Elsevier publication, Volume 1, Number 1, July 2003, pages: 13-64.

[4] F. Ulloa-Vásquez and J .A. Delgado-Penín, "Performance simulation in High Altitude Platforms (Haps) communications systems" IST Project Helinet and Spanish Government (MCYT) TIC2000-0320-CE, supported by the European Commission, available online at: http://www.haps.cl/documentos/13.pdf

[5] M. Hiyama, E. Kulla, T. Oda, M. Ikeda and L. Barolli, "Application of a MANET Testbed for horizontal and vertical scenarios: performance evaluation using delay and jitter metrics" Human-centric Computing and Information Sciences 2011, pages: 1-14.

[6] NEWSKY Project, European Commission Project, "Mobile aeronautical communication network based on internet technologies for cockpit and cabin services integrating satellite and terrestrial data links" German Aerospace Center, Feb. 2007-Oct. 2009, available online at: www.newsky-fp6.eu.

[7] Manual (formerly notes and documentation) of Network Simulator available online at http://www.isi.edu/nsnam/ns/index.html.

[8] TEL-WIMAX-NS2 project documentation, Computer and Electronics Engineering Department at the University of Nebraska-Lincoln, Peter Kiewit Institute in Omaha, Nebraska., (01.10.2012), available online at: http://www.TEL.unl.edu.

[9] "Simulation And Implementation", On-Demand Routing In Multi-Hop Wireless Mobile Ad Hoc Networks, India, (01.10.2012), pages: $73-138$ available online at: http://shodhganga.inflibnet.ac.in/bitstream/10603/4106/1 3/13_chapter\%205.pdf

[10] W. Kiess, M. Mauve, "A survey on real-world implementations of mobile ad-hoc networks" Ad Hoc Networks, Elsevier publication, Volume 5, No. 3, April 2007, pages: 324-339. 